\title{
Susceptibility Based Upon Chemical Interaction with Disease Processes: Potential Implications for Risk Assessment
}

\author{
Gary L. Ginsberg • Rodney R. Dietert • \\ Babasaheb R. Sonawane
}

Published online: 17 October 2014

(C) Springer International Publishing AG 2014

\begin{abstract}
Numerous host and environmental factors may modulate vulnerability and risk. An area of increasing interest is the potential for chemicals to interact with background aging and disease processes, an interaction that may yield cumulative damage, altered chemical potency, and increased disease incidence. We evaluate the interactions possible between chemicals and background disease and identify the type of information needed to evaluate such interactions. Key among these is the existence of a clinically relevant and easy to measure biomarker of disease risk which is also modulated by a particular chemical of interest. This biomarker may be a physiological, biochemical, or genetic indicator that corresponds to a phase of the disease process and indicates where an individual is on the continuum between health and disease. The impact of toxic chemicals on this biomarker can then be used to predict how the chemical modifies disease risk, with this evidence strengthened by additional toxicology and epidemiology data showing toxicant effect on the disease process. Several case studies are presented which describe the toxic chemical, the clinical biomarker, the impacted disease and the evidence that the chemical enhances disease risk: fine particulate matter/decreased heart rate variability/increased cardiopulmonary events; cadmium/ decreased glomerular filtration rate/increased chronic kidney disease; methyl mercury/decreased paraoxonase-1/increased cardiovascular risk; trichloroethylene/increased anti-nuclear antibody/autoimmunity; dioxin/increased CYP1A1/hypertension. These case studies point out that consideration of how a
\end{abstract}

G. L. Ginsberg $(\bowtie)$

Connecticut Department of Public Health, Hartford, CT, USA

e-mail: gary.ginsberg@ct.gov

R. R. Dietert

College of Veterinary Medicine, Cornell University, Ithaca, NY, USA

B. R. Sonawane

National Center for Environmental Assessment, Office of Research and Development, United States Environmental Protection Agency, Washington, DC, USA chemical interacts with background aging and disease processes may increase the public health relevance of risk assessment, identify important vulnerabilities, and provide new ways to calculate risk from exposure to environmental toxicants.

Keywords Risk assessment · Vulnerable subgroups · Particulate matter - Heart rate variability - Cadmium . GFR · Mercury · PON1 · Trichloroethylene · Anti-nuclear antibody - Dioxin - CYP1A1 . Autoimmunity - Cardiovascular disease - Kidney disease $\cdot$ Hypertension

\section{Introduction}

Risk assessment of chemicals has become more concerned with sources of inter-individual variability in recent years as the wide range of host and environmental factors that could potentially affect biological response have been explored. This includes differences related to life stage (fetal, childhood, adult, elderly), gender, lifestyle (exercise, sleep, diet), genetic variation, medications, co-exposure to other chemicals as well as nonchemical stressors, and the existence of endogenous damage processes that lead to disease [1-4]. The idea of cumulative risk assessment is thus being expanded to take into account not only multiple exposure pathways and similarly acting chemicals, but also the range of host-specific factors that may modulate the response to chemical agents. Facilitating this expansion of cumulative risk assessment is the increasing mechanistic understanding of chemical toxicity such that the instigating events (e.g., receptor binding, protein denaturation, DNA damage, lipid peroxidation, other forms of oxidant stress) and the downstream intermediary steps (e.g., genomic and epigenomic expression, disruption of homeostasis, involvement of inflammatory mediators) can be understood to occur to different degrees across the population, depending upon the host factors listed 
above. This paper focuses upon one particular variability factor, the occurrence of disease or pre-disease conditions in the host. As the upstream events that underlie chemical toxicity are compared with the endogenous damage events that underlie disease, their intersection is becoming recognized along with the potential implications for chemical risk assessment. This risk assessment focus was a key recommendation of the National Academy of Sciences as it reviewed the risk assessment methods of the US Environmental Protection Agency (USEPA) in the report referred to as Science and Decisions [4].

Perhaps the most compelling example of the interaction between an environmental agent and background disease is the contribution of fine particulate matter $(\mathrm{PM}<2.5 \mu \mathrm{M})$ to the risk of acute cardiopulmonary mortality. Fine PM can deposit in the deep lung and add to the burden of inflammatory stimuli that promote respiratory dysfunction and cardiac stress. Population studies have repeatedly found a linear or log-linearappearing dose-response curve between fine PM and cardiopulmonary mortality in time series or cross-sectional (across cities) studies [5]. The most plausible explanation for this linearity in response at environmental doses common in the general public is that there is a broad range of vulnerability. Those already having compromised lung function are likely to be at particularly high risk and thus make up the group of lowdose responders [4]. Thus, one implication of toxicant-disease interaction is the continuation of the dose-response curve down to low dose when those with the disease represent a quantitatively important high-sensitivity subgroup. One way to identify such individuals is through upstream biomarkers of disease risk for which certain values (or percentiles of the marker distribution) are known to be indicative of higher disease risk. In the case of fine PM, a pre-disease biomarker of cardiac stress, heart rate variability (HRV) is both a predictive biomarker of risk for major cardiac events and an endpoint that is affected by fine PM [6-8]. While the mechanism(s) for this PM effect are still under investigation, it appears that PM-induced decrease in HRV is a key physiological response that contributes to the imbalance in autonomic control of heart rhythm, underlying, at least in part, cardiovascular morbidity and mortality. The implication is that chemical risk may be expressed as the projected increase in disease through an understanding of how the chemical modulates biomarkers that are predictive of disease risk. This may be particularly relevant at low doses where the chemical on its own might not be able to induce sufficient harm for a measureable outcome but may be a significant contributing factor to the occurrence of disease in susceptible individuals and thus increase population risk for that disease.

\section{Types of Toxicant-Disease Interactions}

Background disease processes may affect vulnerability to toxicant action, while, at the same time, exposure to the toxic chemical may affect the likelihood of disease in a given individual. The manner in which this can occur is described in the following three possible interactions and as outlined in Fig. 1 [9]:

1) The disease alters chemical action by altering its toxicokinetics so as to materially change internal dose. For example, the diabetic state is associated with higher levels of circulating ketones, which induce a particular cytochrome P450 (CYP) called CYP2E1 [10, 11]; this enzyme is known to be involved in the metabolic activation of numerous toxicants (e.g., trichloroethylene [TCE]).

2) The disease weakens host defense mechanisms, as could occur when a pathologic condition is associated with chronic inflammation and oxidative stress.

3) The chemical increases the likelihood of disease by altering pathways that are also affected by the disease process.

In the first two interactions, the presence of the disease process in the exposed individual can be expected to shift the dose-response curve to a lower level dose because the chemical is more effective in the diseased individual. A disease/dose additivity model may be useful in assigning 'dose equivalents' to a particular level of disease. In simple quantitative terms, if $5 \mathrm{mg} / \mathrm{kg} / \mathrm{d}$ of a chemical decreases a disease biomarker by $20 \%$, while aging or a pre-disease state decreases the same biomarker by $10 \%$, one might conclude that the background condition contributes the equivalent of a $2.5 \mathrm{mg} / \mathrm{kg} / \mathrm{d}$ dose. Thus, the starting point for chemical dose response is envisioned to not be zero but some dose equivalent $(2.5 \mathrm{mg} / \mathrm{kg} / \mathrm{d}$ in this case) based upon the level of disease with which it is interacting. An example of this phenomenon occurs with the hepatotoxicity data for the chemical 1,4-dioxane [4]. As shown in Fig. 2, the liver inflammatory response to 1,4-dioxane the pathology term is (hepatic spongiosis) differs greatly between male and female rats, and this corresponds with the gender difference in background rate of spongiosis. The high background of liver inflammation in male rats is additive to the 1,4-dioxane effect. Thus, the defense mechanisms in female rats that act as a barrier to 1,4-dioxane liver toxicity are exceeded in male rats, even in the control animals. In effect, it is as if the background condition is adding chemical dose equivalents such that the starting dose of chemical is already above its threshold for an adverse outcome, making the response at low dose linear in males and threshold in females.

In the third interaction, the chemical increases the likelihood for disease in the population by impacting on toxicity pathways that feed into the disease process. If a common biomarker can be found between disease risk and chemical effect, as in the case of PM affecting HRV, then the impact of the chemical on disease risk may be projected by the degree to 


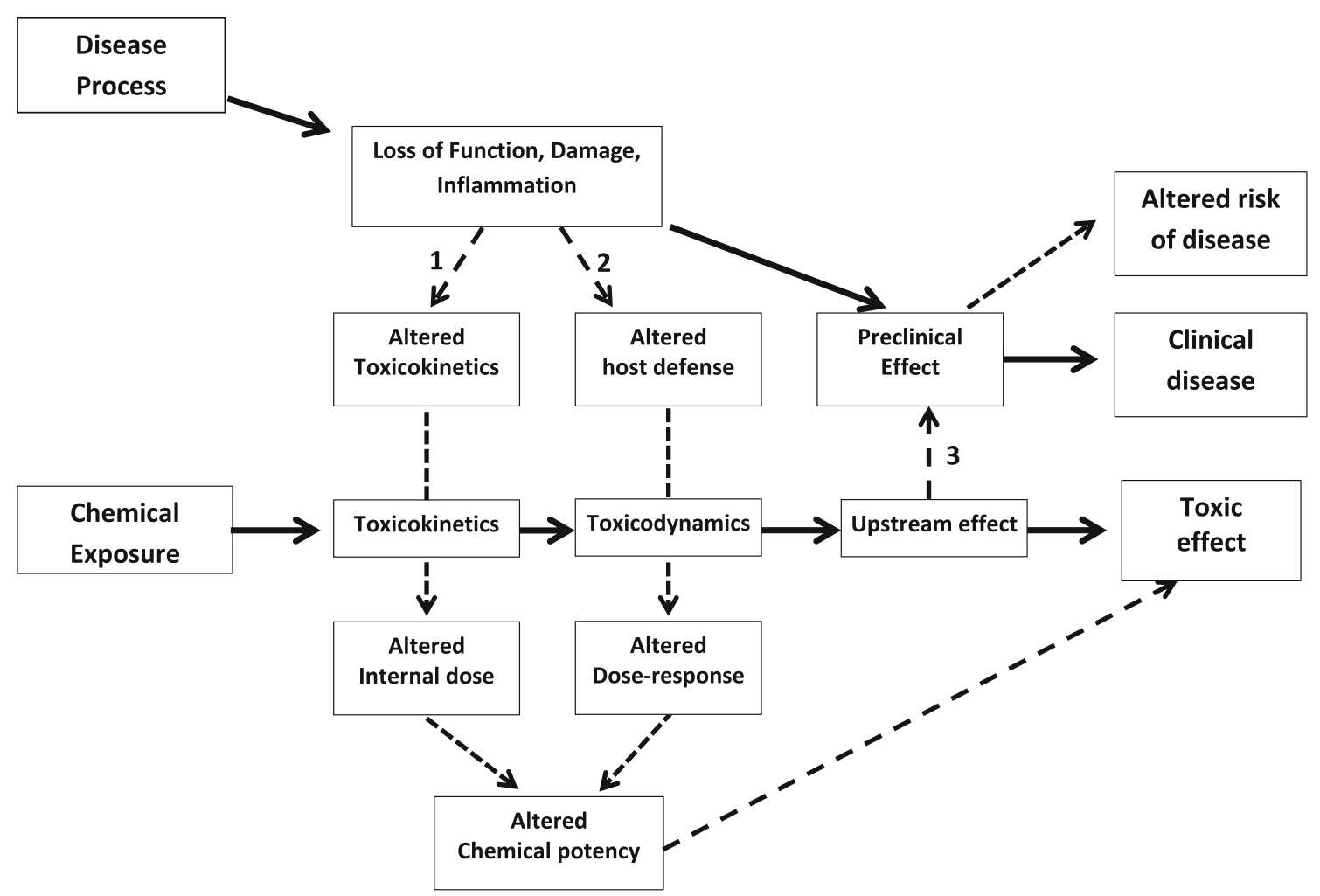

Fig. 1 Potential interaction between chemical exposure and disease process. Solid arrows represent pathways of disease or chemical toxicity, which are typically considered as separate. Dashed arrows show potential interactions numbered as described in text. Interaction could lead to altered chemical potency as the disease process affects host toxicokinetics (Pathway 1) or defense mechanisms (Pathway 2), creating a vulnerability

which it shifts the population towards poor function and increased disease risk. This is depicted in Fig. 3 [4], in which the population distribution for a continuous parameter becomes a vulnerability distribution if the parameter is a biomarker of human disease and if one can assign a degree of risk to a portion of the distribution. In the case of Fig. 3, functional decline increases along the $\mathrm{X}$ axis and crosses a point (vertical line) in which an overt adverse effect or clinical disease can be

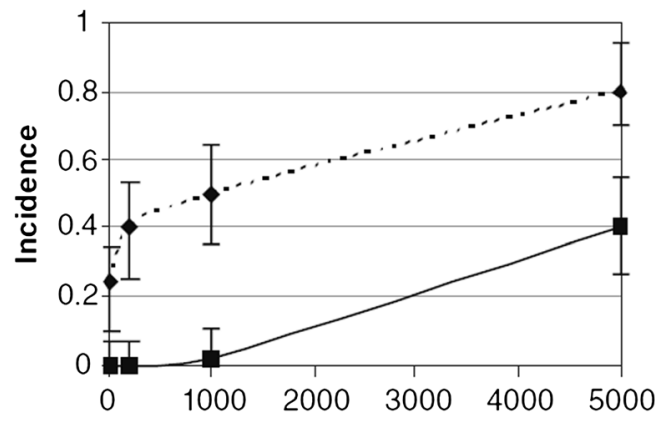

\section{1,4-Dioxane Water Concentration (ppm)}

Fig. 2 Hepatic spongiosis in 1,4-dioxane-exposed rats: influence of control incidence on dose response. Bars indicate the $95 \%$ confidence intervals. Data from Yamazaki et al. [12]; Figure from the National Research Council [4]. Reprinted with permission of National Academies Press, permission \# 04301401 to chemical effect. Interaction could also affect disease risk, especially if the chemical and disease have similar upstream pathways and target organs (Pathway 3). In this case, chemical exposure creates an additional risk factor for disease to occur. Figure modified from the National Research Council report [9], with permission of National Academies Press, permission \# 04301401

recognized. The goal of medical management would be to keep individuals to the left of the clinical threshold, and the goal of risk assessment would be to understand how environmental factors (e.g., chemical and non-chemical stressors) might shift the distribution towards and over the clinical threshold. Thus, chemical risk may be evaluated as the percentage of the population that is shifted into the clinical disease portion of the distribution by a particular dose. This

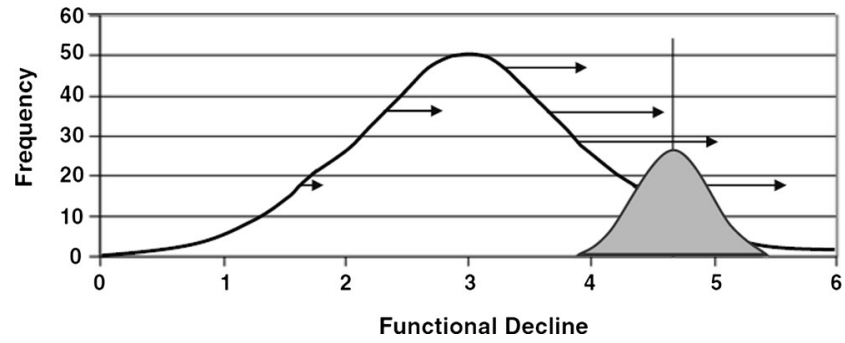

Fig. 3 Population vulnerability distribution in the general case. Arrows represent hypothetical response to the same toxicant dose for people at a given level of functional decline unrelated to the toxicant. The vertical line represents the presumed threshold between overt disease and no adverse effect in median person. The shade area straddling the line represents the distribution of thresholds in the population. Figure from the National Research Council [4]. Reprinted with permission from National Academies Press, permission \# 04301401 
type of risk assessment (modification of disease vulnerability distribution) builds upon the experience with leadinduced shift of population intelligence quotient (IQ) distributions to lower levels, such that even small changes in mean IQ may have particular significance for individuals who are shifted into the low end (low functioning) tail of the IQ distribution [13, 14]. In this example, IQ can be seen as the upstream indicator of neurobehavioral success, as lead-induced IQ lowering has been used to estimate lost earning potential and poorer academic achievement [15]. This approach has recently been exemplified as described in subsequent sections for cadmium and methyl mercury $[16 \bullet \bullet, 17]$.

To study toxicant-disease interactions in this manner, the following needs to be in place:

1) Underlying mechanism: evidence that the mechanism of toxicant action has aspects in common with the disease process (e.g., cellular targets, defense mechanisms, gene expression perturbations).

2) Disease biomarker: predictive biomarker of disease risk is identified for which the population distribution in healthy and diseased individuals (the vulnerability distribution) has been characterized. Shifts in the value of this biomarker are predictive of disease occurrence.

3) Chemical impact on biomarker: the chemical in question impacts the disease biomarker, in a manner which shifts the vulnerability distribution.

4) Chemical impact on disease: supportive epidemiological or toxicological evidence that the chemical can increase risk of the associated disease.

These toxicant-disease interactions are more likely to increase health risk if the rate of the background disease is substantial. With respect to Fig. 3, a rare disease will have a vulnerability distribution with most members of the population far to the left of the clinical threshold. A small shift in the distribution caused by a low dose of toxicant will cause very few (if any) individuals to cross into the clinical disease category - in that case we might say that there is low additivity to background disease. However, for more prevalent diseases, the vulnerability distribution would appear more like that depicted in Fig. 3, with a sizable fraction near the parameter value for clinical effect. In this case, numerous individuals would be sensitive to the toxicant, such that low doses may be able to shift a significant percentage into the disease tail of the distribution [15]. Thus, the increase in risk depends upon the dose response for chemical effect on the parameter and on the background rate of the disease as reflected in the shape of the vulnerability distribution.

The use of an upstream biomarker to evaluate the interaction of a toxicant with a disease process, as exemplified with case studies described below, can be extended to epidemiological research. Studies that test the association between chemical exposure and adverse outcome can evaluate, through an interaction term or nested design, whether that outcome is more or less likely in those with a pre-existing condition (e.g., whether fine PM causes more severe effects in those with preexisting lung disease). While this is useful, often the focus is on quantifying chemical exposure and not the degree of preexisting disease, with the latter often considered in yes/no categorical fashion. Ideally, epidemiology studies would use continuous variables of disease risk (e.g., HRV) in association with continuous variables of chemical exposure to study their interactive effect on adverse outcomes. The case studies described in subsequent sections illustrate the utility of disease risk biomarkers in understanding chemical risk.

\section{Potential Role of Measurement Error}

Before describing specific cases of chemical-disease interaction, it is useful to consider the role of measurement error in epidemiological studies. Evidence for toxicant interaction with background disease comes from epidemiology studies in which linear-appearing slopes exist down to low levels of exposure without evidence of threshold in spite of the likelihood that a threshold exists in individuals [4]. As the example with PM shows, where significant numbers of individuals have pre-existing disease that can be compounded by toxicant exposure, sensitivity and variability is introduced into the population dose response that tends to linearize the response at low doses. However, it has been argued that measurement error, rather than variability in response and interaction with background disease, is the reason why fine PM has a linearappearing dose-response curve $[18,19]$. Measurement error tends to smooth out or blur the dose response so that it can be more difficult to detect a clear break (non-linearity or threshold) in the dose-response curve. However, measurement error can also weaken associations and decrease the significance of regression coefficients such that those studies in which the regression coefficients are significant may be because they had less measurement error (or other confounders). There are other reasons to lessen emphasis on the measurement error explanation for linear-appearing dose response in population epidemiology studies.

A cornerstone of the measurement error hypothesis is the Brauer et al. [20] 2002 demonstration that errors in PM measurements are capable of washing out a built-in threshold in PM dose response for mortality. Brauer et al. [20] took personal monitoring measurements for 16 individuals for both PM and sulphate and compared these measurements with pollution measurements at regional monitors. The PM measurement error was large when relying on central monitoring locations, indicating that local and personal factors play an important role 
in the value obtained. Brauer et al. [20] further demonstrated that the degree of measurement error in their PM data was sufficient to smooth out the dose response and obscure a threshold they had built into their dose-response model for PM-induced mortality [20]. Rhomberg and colleagues [18, 19] reviewed the cases of PM, nitrogen oxide, and ozone measurement variability with respect to the potential for measurement error to obscure thresholds in air pollution epidemiology studies. However, they do not consider the example of sulphate pollution, even though this is a key part of the Brauer et al. [20] study. In contrast to the PM results, sulphate measures from personal monitors correlated quite well with central outdoor air monitors across the 16 subjects. This led Brauer et al. [20] to show that, given the small measurement error for sulphate, it is easy to show a threshold in population studies should one exist (they simulated three different thresholds, each reliably reproduced for sulphate when the sulphate measurement error was built into the model). This suggests measurement error should not substantially mask population thresholds for sulphate or sulphur dioxide, for which total sulphate is a surrogate measure. By examining the population dose response for sulphate-related mortality relied upon by Brauer et al. (2002) [21], and an update of that in the World Health Organisation (WHO) guideline of 2005 [22], it is clear that many sulphate population studies are associated with linearappearing dose response and that "As with ozone and PM, no obvious threshold levels have so far been identified in these population-based studies" [22]. According to the Brauer et al. [20] 2002 analysis, measurement error is unlikely to explain the lack of threshold for sulphur dioxide-increased mortality in the population studies reviewed by WHO. The sulphate example makes the case that factors other than measurement error can cause a linear dose response down to low dose in population studies for inhaled irritants. These other factors (e.g., inter-human variability) are summarized above for the general case.

Beyond air pollutants, numerous examples exist of population studies in which linear slopes occur down to low levels of exposure without evidence of threshold. In many of these cases, measurement error is unlikely to explain the lack of threshold since the index of exposure is biomonitoring data, i.e., quantitative internal evidence of exposure that does not rely upon extrapolation from job histories or external measurements. Examples include linear-appearing slopes for arsenic-induced cancer and non-cancer effects in relation to urinary inorganic arsenic [9]; for mercury-induced neurodevelopmental deficits in relation to maternal hair mercury $[23,24]$, which has been modelled as a linear relationship by Axelrad et al. [25] (2007); cadmium-induced decline in glomerular filtration rate (GFR), which has been found to have significant linear regression to low levels of urinary cadmium [26]; and lead-induced neurodevelopmental deficits in relation to childhood blood lead [27]. Thus, while it is important to be aware of the potential influence of measurement error on dose response in human population studies, the examples mentioned above indicate that measurement error is unlikely to explain the linear-appearing slopes in many epidemiology studies and that it is important to recognize the role of background disease processes and other sources of intrahuman variability in extending the response to low dose.

\section{Case Studies of Toxicant-Disease Interaction}

As shown in Table 1, the elements for analyzing the toxicant-disease interaction are in place for at least three case studies (fine PM - discussed above, cadmium, and methyl mercury - discussed below) and are plausible for several others as discussed below. The biomarkers listed in the table facilitate exploration of these interactions, although their accuracy and link to clinical disease require assessment when considering their use in risk assessment.

\section{Cadmium-Induced Renal Toxicity and the Risk of Chronic} Kidney Disease

Kidney function progressively declines with age, as measured by various indices, including GFR [33]. A variety of conditions and diseases can put additional stress on the kidney, leading to increased risk of kidney disease. These conditions include diabetes, hypertension, obesity, cardiovascular disease, and systemic lupus erythematosus (SLE). The medical definition of advanced (stage 3 chronic kidney disease [CKD]) is based solely upon GFR: a GFR less than $60 \mathrm{ml} /$ $\min / 1.73 \mathrm{~m}^{2}$ for at least 3 months is diagnostic of CKD. This GFR is well below the normal adult rate and is considered indicative of CKD regardless of age or the existence of other changes to the kidney [34]. Stage 3 CKD is clinically important, as it often leads to more serious renal impairment and the potential need for dialysis.

Certain environmental toxicants may interact with background aging and disease processes and jointly affect kidney function. Cadmium is a toxic metal that, by virtue of its known localization in the kidney, has this as a prominent target organ. Upstream oxidative stress signaling induced by cadmium (elevated mitogen-activated protein kinase [MAPK]-38 in renal cells) as well as clinical markers of cadmium toxicity (protein leakage into urine, decreased GFR) show that the effects of cadmium on the kidney are similar to that which occur from aging and disease [35-37]. The potential interaction of cadmium with background aging and disease processes has been assessed based upon modulation of the population distribution of GFR and the resulting increased risk of CKD [16••]. The potency for cadmium-induced decrease in GFR was derived from the regression slope observed in a study of 
Table 1 Chemicals, diseases, and biomarkers that can be used to evaluate toxicant-disease interaction

\begin{tabular}{llll}
\hline Chemical or physical agent & Interacting disease & Disease biomarker & Chemical impact on disease biomarker \\
\hline Fine particulate matter & Cardiopulmonary mortality & HRV & Decrease in HRV [6] \\
Cadmium & Renal disease & GFR & Decrease in GFR [26] \\
Methyl mercury & Acute cardiac events & PON1 & Decrease in PON1 [28] \\
Trichloroethylene & Autoimmune disease & ANAs & Increase in ANAs [29] \\
TCDD, PAHs & Hypertension & CYP1A1 & Increase in CYP1A1 [30] \\
Styrene, ethylene oxide, radiation & Cancer, diabetes, heart disease, renal disease & CBMN & Increase in CBMN [31, 32] \\
\hline
\end{tabular}

$A N A s$ anti-nuclear antibodies, $C B M N$ cytokinesis-blocked micronucleus, $C Y P$ cytochrome $\mathrm{P} 450, G F R$ glomerular filtration rate, $H R V$ heart rate variability, $P A H s$ polycyclic aromatic hydrocarbons, $P O N 1$ paraoxonase-1

816 Swedish women chronically exposed to low environmental (rather than occupational) doses of cadmium [26]. This cadmium effect was applied to the GFR population distribution found in healthy adult women, resulting in a leftward shift of the distribution and inducing a greater percentage of the population to fall below the clinical definition of stage $3 \mathrm{CKD}$ $\left(60 \mathrm{ml} / \mathrm{min} / 1.73 \mathrm{~m}^{2}\right)$. The analysis showed that environmentally relevant cadmium doses of $0.1-1 \mu \mathrm{g} / \mathrm{kg} / \mathrm{d}$ were estimated to increase the risk for CKD by $3-30 \%[16 \bullet \bullet$. This example demonstrates that toxicant-induced risk of organ damage can be put in the context of population risk of chronic disease when the toxicant impacts an upstream biomarker that is predictive of chronic disease. This provides a quantitative method to assess the interaction of toxicant with aging and disease processes and thus may provide risk estimates of greater relevance to public health.

\section{Methyl Mercury-Induced Oxidative Stress and the Risk of Cardiovascular Events}

Cardiovascular disease is related to numerous risk factors ranging from genetic predisposition to dietary composition, activity level, stress and related disease states (e.g., metabolic disorder, thyroid imbalance, renal disease, and hypertension). In most cases, the potential role of toxic chemicals is overlooked. In the case of methyl mercury (MeHg), several epidemiology studies support an increased risk for cardiovascular disease, both in terms of narrowing of blood vessels and in increasing the risk of acute cardiac events [38-40]. However, not all epidemiology studies support such a link between $\mathrm{MeHg}$ and cardiovascular risk [41, 42]. This inconsistency may occur because $\mathrm{MeHg}$ exposure stems primarily from fish ingestion, and fish contain cardioprotective components, the most notable being omega-3 fatty acids [43, 44]. Therefore, the net cardiovascular risk or benefit associated with fish consumption is expected to vary depending upon which fish species and amounts are consumed by the population studied.

Further evidence of a cardiovascular risk associated with $\mathrm{MeHg}$ is the ability of $\mathrm{MeHg}$ to impair a cardioprotective enzyme known as paraoxonase-1 (PON1). PON1 is associated with high-density lipoprotein (HDL) and prevents the oxidation of serum lipids and thus also the accumulation of oxidized lipid in vascular walls [45-47]. Its utility as a biomarker of cardiovascular risk has been demonstrated in several studies, including one in which 1,399 middle- to older-aged men were followed for 3 years after baseline serum PON1 measurements were taken [48]. The elevated risk of acute cardiovascular events in relation to baseline PON1 allowed the construction of a PON1 vulnerability distribution for cardiovascular risk [17]. Mercury has been shown to inhibit PON1 activity both in vitro and in vivo, with the in vivo study of a native fisheating population in Canada demonstrating a dose response for lowering serum PON1 levels [28]. The mercury effect on PON1 likely stems from its attack on the lone sulfhydryl of PON1, a structural feature that is critical to its antioxidant properties. Application of the MeHg dose response for inhibition of serum PON1 to this PON1 vulnerability distribution led to a shift to lower levels of PON1 and to a greater number of individuals in the high-risk categories. This effect was accentuated when taking into account PON1 polymorphisms that lower the activity of the enzyme and thus have a compounding effect with $\mathrm{MeHg}$ on enzyme level and cardiovascular risk. Quantitation of the risk from an environmentally relevant MeHg dose of $0.3 \mu \mathrm{g} / \mathrm{kg} / \mathrm{d}$ led to a projected decrease in PON1 by $6.1 \%$ and an increase in population risk of acute cardiovascular events by $9.7 \%$ [17]. Thus, reliance on mechanistic considerations and an upstream biomarker of cardiovascular risk enabled a quantitative investigation of the potential impact of MeHg on public health in a manner that complements the epidemiological evidence in this area.

\section{Trichloroethylene-Induced Autoimmunity}

Autoimmunity encompasses an array of progressive debilitating diseases that affect numerous organs, including the kidney, liver, thyroid, pancreas, gastrointestinal tract, skin, connective tissues, nervous system, and lung. The common link is inappropriate direction of the immune system against host tissues, which leads to chronic inflammation. This may result from the 
modification of host proteins so that they become recognized as foreign, access to tissue components that are normally hidden but become exposed to immune cells because of cellular damage, or perturbation of immune regulation such that there is inadequate suppression of 'self-reactive' $\mathrm{T}$ and $\mathrm{B}$ cells $[49,50]$. Exposure to chemicals may contribute to autoimmunity via these mechanisms, as some chemicals are capable of modifying host proteins to render them immunogenic [51], while other chemicals may damage tissues or alter gene regulation to affect the degree to which the immune system is self-reactive. Numerous pharmacological agents and environmental toxicants are implicated as promoting autoimmune conditions [52-54], with perhaps the best recognized of these being TCE [29]. TCE-induced autoimmunity has been evidenced in several mouse models, including one for SLE [55, 56], and in at least one strain not known for autoimmunity (B6C3F1 mice) [57]. Human studies also provide evidence of autoimmunity, as workers exposed to TCE had increased occurrence of scleroderma [58-60] and several other immune-related conditions [29]. The mechanism(s) for TCEinduced autoimmunity is unknown but may involve an initial toxic reaction in the liver involving TCE metabolism via CYP2E1 and subsequent oxidative damage and chronic hepatitis that stimulates the immune system to react against the liver and other tissues [61, 62]. Supplementation with the antioxidant sulfhydryl $\mathrm{N}$-acetylcysteine was able to prevent TCE-related liver damage and autoimmunity [63]. This mechanism is likely to be operative at the high doses used in the Wang et al. [62, 63] studies $(1,300 \mathrm{mg} / \mathrm{kg}$ via intraperitoneal injection) with this and perhaps other mechanisms occurring at lower doses where TCE autoimmunity has also occurred. TCE induces protein adducts in mouse liver, making it possible that the dichloroacetylated lysine adduct associated with TCE is a source of immunogenicity at low dose, although this possibility has not been fully explored [64]. While doseresponse studies are thus far limited, the lowest dose found to enhance autoimmune parameters was $0.35 \mathrm{mg} / \mathrm{kg} / \mathrm{d}$ in drinking water both in the mouse SLE model and in B6C3F1 mice $[58,65 \bullet \cdot$. This dose was associated with serological evidence of TCE-induced autoimmunity (anti-nuclear antibody [ANA] titers) with some evidence of renal involvement [58]. It also appears that inorganic mercury can enhance TCE-induced autoimmunity in autoimmune-prone mice, although the mechanism for this interaction has not been explored [66].

To better understand TCE-induced risk of autoimmunity at environmentally relevant doses, a biomarker of autoimmunity risk that is also sensitive to TCE would be needed. A potential candidate is ANA, since elevated ANA titer is a common finding in TCE studies in both mice and humans, with this effect extending down to the lowest doses tested [29, 65••]. Antibodies directed against nuclear elements and other proteins is a serologic marker in a variety of autoimmune conditions, with this being used to assist early diagnosis of conditions such as type 1 diabetes and SLE $[67,68]$. Thus, it is plausible that TCE-induced elevation of serum autoantibody markers can combine with genetic predisposition and inflammatory disease processes to elevate the risk of autoimmune conditions. Studies that further explore the dose response for TCE-induced increases in ANA and other autoantibodies in autoimmune disease models may help clarify the degree to which TCE increases the risk for these conditions in humans.

\section{Dioxins and Other Aryl Hydrocarbon Receptor Ligands} in Relation to Blood Pressure

Hypertension is a multifactorial condition that is highly prevalent in the human population. The fact that several epidemiological studies have shown a link between the expression of CYP1A1, a CYP isozyme that is regulated by the aryl hydrocarbon (Ah) receptor, and hypertension suggest that stimulation of the Ah receptor may contribute to the occurrence of hypertension. For example, epidemiological studies suggest that exposure to dioxin-like compounds is associated with higher blood pressure and that the precursor effect of the prototypical dioxin, TCDD, is ablated in knockout mice that lack the CYP1A1 gene [69]. Further, polymorphisms in CYP1A1 that modulate its inducibility and expression have corresponding effects on the risk for ischemic heart disease $[70,71]$. The mechanistic link between CYP1A1 expression and hypertension appears to be related to increased reactive oxygen generation as a byproduct of $\mathrm{Ah}$ receptor expression and CYP1A1 metabolic processes $[69,72]$. However, other aspects of $\mathrm{Ah}$ receptor activation by environmental ligands may also promote the hypertensive state as knockout mouse studies have shown the importance of the $\mathrm{Ah}$ receptor to maintaining blood pressure stability [73]. The contribution of Ah receptor ligands such as TCDD and polycyclic aromatic hydrocarbons (PAHs) to hypertensive and ischemic risk in human populations is becoming increasingly recognized $[74,75]$. Thus, a potential biomarker of hypertension risk and chemical-disease interaction may be Ah receptor activation in the form of CYP1A1 expression. It may be possible to screen the expression of, and polymorphisms in, this candidate biomarker in easily accessible samples such as peripheral blood lymphocytes (PBLs) as an indicator of the CYP1A1 status of cardiac and endothelial tissues [76]. Further studies of the relationship between CYP1A1 expression and blood pressure that control for the array of factors that can modulate blood pressure are needed to facilitate the use of CYP1A1 as a marker of hypertension risk in the general population. 
Induction of Micronuclei by Pro-Oxidant Chemicals in Relation to Cancer and Inflammatory Disease

Oxidative stress is a result of inflammatory processes that underlie many common diseases. Exposure to pro-oxidant chemicals can add to the systemic level of oxidative stress and, at least in theory, also add to inflammation and disease burden. Oxidative stress can be biomonitored in various ways, with the detection of micronuclei, a clastogenic result of oxidant attack on DNA, becoming increasingly common $[32,77]$. Other agents that damage DNA via the formation of adducts or strand breakage can also lead to micronuclei formation, e.g., styrene oxide, ethylene oxide, residential radon, sunlight, and chronic alcohol intake [31, 78-81]. This form of DNA damage is readily seen in a cytokinesis-blocked micronucleus assay (CBMN) in which PBLs are arrested during cell division with cytochalasin $\mathrm{B}$ to freeze the cell in the binucleated state, during which it is easy to detect chromosome damage in the form of micronuclei. Increased rates of CBMN have been found in PBLs in association with age [82], and a meta-analysis of relevant epidemiology studies shows increased population cancer risk in individuals with moderately and highly elevated CBMN rates [83]. Other diseases are also marked by increased CBMN rates, including renal disease [84], type 2 diabetes [85], and cardiovascular disease [86••]. The predictive value of CBMN in determining cardiovascular risk is shown in Fig. 4, in which subjects with higher CBMN scores at the beginning of the study had greater rates of cardiac events and cardiovascular mortality upon follow-up [86••]. Thus, CBMN in PBLs may be a convenient marker of systemic oxidative stress and risk for a variety of chronic diseases. Given the wide variety of chemicals that can induce oxidative stress, CBMN may be a useful effect biomarker that can integrate across chemical and non-chemical stressors to

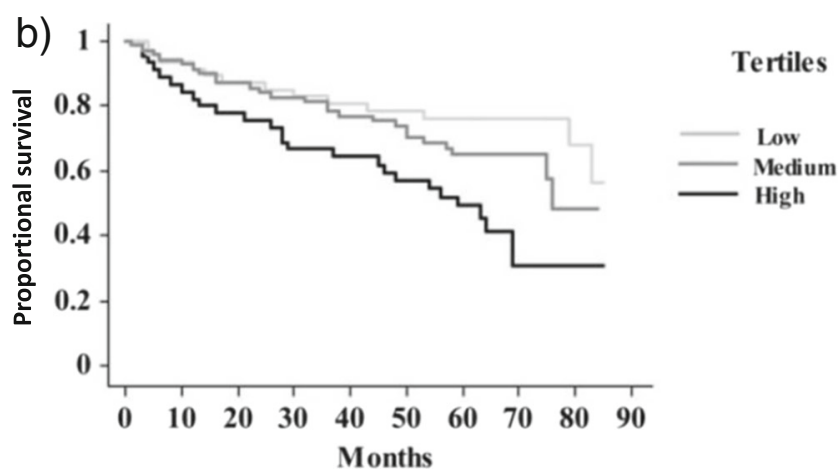

Fig. 4 Predictive value of a cytokinesis-blocked micronucleus assay assay for cardiovascular survival. Kaplan-Meier curves showing cumulative rates of survival for cardiovascular mortality for major adverse events in patients with coronary artery disease according to micronucleus assay at baseline. Figure from Andreassi et al. [86••]. Reprinted with permission from Oxford University Press, license agreement \# 3378870482866 indicate cumulative oxidative stress. Identification of oxidant chemicals that can, at low dose, shift the CBMN population distribution to higher scores will help prioritize those agents that are likely to make a substantial contribution to the background rate of CBMN and thus affect population risk of chronic disease.

\section{Summary and Conclusions}

The increasing focus on cumulative risk is helping risk assessors explore factors that would normally be treated with default uncertainty factors or not be addressed at all. An important variability factor concerns chemical interaction with background aging and disease processes. Disease processes that are prevalent in the population may confer a different vulnerability to toxicant action and may provide opportunities for toxicants to be active at low dose, levels below those representing a threshold in the average healthy individual. By developing a vulnerability distribution for disease occurrence based upon highly specific well validated clinically recognized biomarkers, it is feasible to explore how a chemical may interact with the disease process and shift the biomarker distribution to impact the risk of disease. The case examples involving cadmium/GFR and methyl mercury/ PON1 provide quantitative examples of this approach, while the possibility for such analyses of TCE-induced autoimmunity and dioxin-induced hypertension may lead to better understanding of the public health risks from these chemicals. The CBMN example highlights that a non-specific biomarker of systemic oxidative stress may be useful as a cumulative index of disease risk and the contribution from individual pro-oxidant chemicals. To facilitate these and additional interaction studies, it will be critical for medical researchers, clinicians, toxicologists, and epidemiologists to jointly identify biomarkers of disease risk and explore how toxicants may perturb these biomarkers and thus modify population risk.

Disclaimer The authors declare that they have no competing financial interests. The views expressed in this paper are those of the authors and do not necessarily reflect the views and/or policies of the State of Connecticut, the US Environmental Protection Agency, or Cornell University.

\section{Compliance with Ethics Guidelines}

Conflict of Interest Gary L. Ginsberg, Rodney R. Dietert, and Babasaheb R. Sonawane declare that they have no conflicts of interest.

Human and Animal Rights and Informed Consent This article does not contain any studies with human or animal subjects performed by any of the authors. 


\section{References}

Papers of particular interest, published recently, have been highlighted as:

- Of major importance

1. Faustman EM, Silbernagel SM, Fenske RA, Burbacher TM, Ponce RA. Mechanisms underlying children's susceptibility to environmental toxicants. Environ Health Perspect. 2000;108 Suppl 1:13-21.

2. Ginsberg G, Hattis D, Russ A, Sonawane B. Pharmacokinetic and pharmacodynamic factors that can affect sensitivity to neurotoxic sequelae in elderly individuals. Environ Health Perspect. 2005;113: 1243-9.

3. Ginsberg GL, Asgharian B, Kimbell JS, Ultman JS, Jarabek AM. Modeling approaches for estimating the dosimetry of inhaled toxicants in children. J Toxicol Environ Health A. 2008;71:166-95.

4. National Research Council. Science and decisions: advancing risk assessment. Washington DC: National Academy Press; 2009.

5. Schwartz J, Coull B, Laden F, Ryan L. The effect of dose and timing of dose on the association between airborne particles and survival. Environ Health Perspect. 2008;116:64-9.

6. Nyhan M, McNabola A, Misstear B. Comparison of particulate matter dose and acute heart rate variability response in cyclists, pedestrians, bus and train passengers. Sci Total Environ. 2014;468 469:821-31.

7. Ramirez-Villegas JF, Lam-Espinosa E, Ramirez-Moreno DF, Calvo-Echeverry PC, Agredo-Rodriguez W. Heart rate variability dynamics for the prognosis of cardiovascular risk. PLoS ONE. 2011;6:e17060.

8. Colhoun HM, Francis DP, Rubens MB, Underwood SR, Fuller JH. The association of heart-rate variability with cardiovascular risk factors and coronary artery calcification: a study in type 1 diabetic patients and the general population. Diabetes Care. 2001;24:110814.

9. National Research Council. Critical aspects of EPA's IRIS assessment of inorganic arsenic: interim report. Washington DC: National Academy Press; 2014.

10. Arinç E, Arslan S, Adali O. Differential effects of diabetes on CYP2E1 and CYP2B4 proteins and associated drug metabolizing enzyme activities in rabbit liver. Arch Toxicol. 2005;79:427-33.

11. Suhua W, Rongzhu L, Wenrong X, Guangwei X, Xiaowu Z, Shizhong W, et al. Induction or inhibition of cytochrome P450 2E1 modifies the acute toxicity of acrylonitrile in rats: biochemical evidence. Arch Toxicol. 2010;84:461-9.

12. Yamazaki K, Ohno H, Asakura M, Narumi A, Ohbayashi H, Fujita H, Ohnishi M, Katagiri T, Senoh H, Yamanouchi K, Nakayama E, Yamamoto S, Noguchi T, Nagano T, Enomoto M, Sakabe H. Twoyear toxicological and carcinogenesis studies of 1, 4-dioxane in F344 rats and BDF1 mice: Drinking studies. Pp. 193-198 in Proceedings of the Second Asia-Pacific Symposium on Environmental and Occupational Health, 22-24 July, 1993, Kobe, Japan, K. Sumino, and S. Sato, eds. Kobe: International Center for Medical Research Kobe, University School of Medicine. 1994.

13. Schwartz J. Societal benefits of reducing lead exposure. Environ Res. 1994;66:105-24.

14. Bellinger DC. Interpretation of small effect sizes in occupational and environmental neurotoxicology: individual versus population risk. Neurotoxicology. 2007;28:245-51.

15. Salkever DS. Assessing the IQ-earnings link in environmental lead impacts on children: have hazard effects been overstated? Environ Res. 2014;131:219-30.
16.• Ginsberg GL. Cadmium risk assessment in relation to background risk of chronic kidney disease. J Toxicol Environ Health A. 2012;75:374-90. This study provides the logic and methodology for chemical-disease interaction analysis within a risk-assessment context.

17. Ginsberg G, Sonawane B, Nath R, Lewandowski P. Paraoxonase-1 (PON1) as disease biomarker for chemical-induced cardiovascular risk: the example of methyl mercury. J Toxicol Environmental Health A. 2014;77:1004-1023.

18. Rhomberg LR, Chandalia JK, Long CM, Goodman JE. Measurement error in environmental epidemiology and the shape of exposure-response curves. Crit Rev Toxicol. 2011;41:651-71.

19. Rhomberg LR, Goodman JE, Haber LT, Dourson M, Andersen ME, Klaunig JE, et al. Linear low-dose extrapolation for noncancer health effects is the exception, not the rule. Crit Rev Toxicol. 2011;41:1-19.

20. Brauer M, Brumm J, Vedal S, Petkau AJ. Exposure misclassification and threshold concentrations in time series analyses of air pollution health effects. Risk Anal. 2002;22:1183-93.

21. World Health Organization. Air Quality Guidelines. 1999 Available at http://htee.meng.auth.gr/AIR-EIA/METHODS/ AQGuide/AQGuide.htm. WHO, Geneva. Accessed 20 April 2014.

22. World Health Organization. Air Quality Guidelines. 2005. Chapter 13: Sulfur Oxides, pp 395-417. WHO, Geneva. Available at http://www.euro.who.int/_data/assets/pdf_file/0005/78638/ E90038.pdf. Accessed 20 April 2014.

23. USEPA. IRIS File for Methyl Mercury. 2001. Available at http:// www.epa.gov/iris/subst/0073.htm. Accessed 20 April 2014.

24. National Research Council. The toxicological effects of methyl mercury. Washington DC: National Academy Press; 2000.

25. Axelrad DA, Bellinger DC, Ryan LM, Woodruff TJ. Dose-response relationship of prenatal mercury exposure and IQ: an integrative analysis of epidemiologic data. Environ Health Perspect. 2007;115: 609-15.

26. Akesson A, Lundh T, Vahter M, Bjellerup P, Lidfeldt J, Nerbrand C, et al. Tubular and glomerular kidney effects in Swedish women with low environmental cadmium exposure. Environ Health Perspect. 2005;113:1627-31.

27. Bellinger DC. Very low lead exposures and children's neurodevelopment. Curr Opin Pediatr. 2008;20:172-7.

28. Ayotte P, Carrier A, Ouellet N, Boiteau V, Abdous B, Sidi EA, et al. Relation between methylmercury exposure and plasma paraoxonase activity in Inuit adults from Nunavik. Environ Health Perspect. 2011;119:1077-83.

29. Cooper GS, Makris SL, Nietert PJ, Jinot J. Evidence of autoimmune-related effects of trichloroethylene exposure from studies in mice and humans. Environ Health Perspect. 2009;117: 696-702.

30. Kopf PG, Walker MK. 2,3,7,8-tetrachlorodibenzo-p-dioxin increases reactive oxygen species production in human endothelial cells via induction of cytochrome P4501A1. Toxicol Appl Pharmacol. 2010;245:91-9.

31. Migliore L, Naccarati A, Coppedè F, Bergamaschi E, De Palma G, Voho A, et al. Cytogenetic biomarkers, urinary metabolites and metabolic gene polymorphisms in workers exposed to styrene. Pharmacogenet Genomics. 2006;16:87-99.

32. Bolognesi C, Fenech M. Micronucleus assay in human cells: lymphocytes and buccal cells. Methods Mol Biol. 2013;1044:191-207.

33. Oh SW, Kim S, Na KY, Kim KW, Chae DW, Chin HJ. Glomerular filtration rate and proteinuria: association with mortality and renal progression in a prospective cohort of a community-based elderly population. PLoS ONE. 2014;9:e94120.

34. National Kidney Foundation. KDOQI Clinical Practice Guidelines for Chronic Kidney Disease: Evaluation, Classification, and Stratification. Part 4: Definition and classification of stages of chronic kidney disease. 2002. Available at http://www.kidney.org/ 
professionals/kdoqi/guidelines_ckd/p4_class_g1.htm. Accessed 20 April 2014.

35. Iwatsuki M, Inageda K, Matsuoka M. Cadmium induces phosphorylation and stabilization of c-Fos in HK-2 renal proximal tubular cells. Toxicol Appl Pharmacol. 2011;251:209-16.

36. Hirano S, Sun X, DeGuzman CA, Ransom RF, McLeish KR, Smoyer WE, et al. p38 MAPK/HSP25 signaling mediates cadmium-induced contraction of mesangial cells and renal glomeruli. Am J Physiol Renal Physiol. 2005;288:F1133-43.

37. Grande MT, López-Novoa JM. Therapeutical relevance of MAPkinase inhibitors in renal diseases: current knowledge and future clinical perspectives. Curr Med Chem. 2008;15:2054-70.

38. Houston MC. Role of mercury toxicity in hypertension, cardiovascular disease, and stroke. J Clin Hypertens. 2011;13:621-7.

39. Virtanen JK, Voutilainen S, Rissanen TH, Mursu J, Tuomainen TP, Korhonen MJ, et al. Mercury, fish oils, and risk of acute coronary events and cardiovascular disease, coronary heart disease, and allcause mortality in men in eastern Finland. Arterioscler Thromb Vasc Biol. 2005;25:228-33.

40. Salonen JT, Seppänen K, Lakka TA, Salonen R, Kaplan GA. Mercury accumulation and accelerated progression of carotid atherosclerosis: a population-based prospective 4-year follow-up study in men in eastern Finland. Atherosclerosis. 2000;148:265-73.

41. Mozaffarian D, Shi P, Morris JS, Spiegelman D, Grandjean P, Siscovick DS, et al. Mercury exposure and risk of cardiovascular disease in two U.S. cohorts. N Engl J Med. 2011;364:1116-25.

42. Hallgren CG, Hallmans G, Jansson JH, Marklund SL, Huhtasaari F, Schütz A, et al. Markers of high fish intake are associated with decreased risk of a first myocardial infarction. Br J Nutr. 2001;86: 397-404.

43. Mozaffarian D, Rimm EB. Fish intake, contaminants, and human health: evaluating the risks and the benefits. JAMA. 2006;296: 1885-99.

44. Mahaffey KR, Sunderland EM, Chan HM, Choi AL, Grandjean P, Mariën K, et al. Balancing the benefits of n-3 polyunsaturated fatty acids and the risks of methylmercury exposure from fish consumption. Nutr Rev. 2011;69:493-508.

45. Mackness B, Quarck R, Verreth W, Mackness M, Holvoet P. Human paraoxonase-1 overexpression inhibits atherosclerosis in a mouse model of metabolic syndrome. Arterioscler Thromb Vasc Biol. 2006;26:1545-50.

46. Hine D, Mackness B, Mackness M. Coincubation of PON1, APO $\mathrm{A} 1$, and LCAT increases the time HDL is able to prevent LDL oxidation. IUBMB Life. 2012;64:157-61.

47. Macharia M, Hassan MS, Blackhurst D, Erasmus RT, Matsha TE. The growing importance of PON1 in cardiovascular health: a review. J Cardiovasc Med. 2012;13(7):443-53.

48. Bhattacharyya T, Nicholls SJ, Topol EJ, Zhang R, Yang X, Schmitt $\mathrm{D}$, et al. Relationship of paraoxonase 1 (PON1) gene polymorphisms and functional activity with systemic oxidative stress and cardiovascular risk. JAMA. 2008;299:1265-76.

49. Zohar Y, Wildbaum G, Karin N. Beneficial autoimmunity participates in the regulation of rheumatoid arthritis. Front Biosci. 2006;11:368-79.

50. Rose NR. Mechanisms of autoimmunity. Semin Liver Dis. 2002;22:387-94.

51. Pumford NR, Martin BM, Thomassen D, Burris JA, Kenna JG, Martin JL, et al. Serum antibodies from halothane hepatitis patients react with the rat endoplasmic reticulum protein ERp72. Chem Res Toxicol. 1993;6:609-15.

52. D'Cruz D. Autoimmune diseases associated with drugs, chemicals and environmental factors. Toxicol Lett. 2000;112-113:421-32.

53. Hess EV. Environmental chemicals and autoimmune disease: cause and effect. Toxicology. 2002;181-182:65-70.

54. Barragán-Martínez C, Speck-Hernández CA, Montoya-Ortiz G, Mantilla RD, Anaya JM, Rojas-Villarraga A. Organic solvents as risk factor for autoimmune diseases: a systematic review and metaanalysis. PLoS ONE. 2012;7(12):e51506.

55. Blossom SJ, Doss JC, Hennings LJ, Jernigan S, Melnyk S, James SJ. Developmental exposure to trichloroethylene promotes CD4+ T cell differentiation and hyperactivity in association with oxidative stress and neurobehavioral deficits in MRL $+/+$ mice. Toxicol Appl Pharmacol. 2008;231:344-53.

56. Cai P, König R, Boor PJ, Kondraganti S, Kaphalia BS, Khan MF, et al. Chronic exposure to trichloroethene causes early onset of SLE-like disease in female MRL $+/+$ mice. Toxicol Appl Pharmacol. 2008;228:68-75.

57. Keil DE, Peden-Adams MM, Wallace S, Ruiz P, Gilkeson GS. Assessment of trichloroethylene (TCE) exposure in murine strains genetically-prone and non-prone to develop autoimmune disease. $\mathrm{J}$ Environ Sci Health A Toxic Hazard Subst Environ Eng. 2009;44: 443-53.

58. Diot E, Lesire V, Guilmot JL, Metzger MD, Pilore R, Rogier S, et al. Systemic sclerosis and occupational risk factors: a casecontrol study. Occup Environ Med. 2002;59:545-9.

59. Garabrant DH, Lacey Jr JV, Laing TJ, Gillespie BW, Mayes MD, Cooper BC, et al. Scleroderma and solvent exposure among women. Am J Epidemiol. 2003;157:493-500.

60. Nietert PJ, Sutherland SE, Silver RM, Pandey JP, Knapp RG, Hoel $\mathrm{DG}$, et al. Is occupational organic solvent exposure a risk factor for scleroderma? Arthritis Rheum. 1998;41:1111-8.

61. Griffin JM, Gilbert KM, Pumford NR. Inhibition of CYP2E1 reverses $\mathrm{CD} 4+\mathrm{T}$-cell alterations in trichloroethylene-treated MRL+/+mice. Toxicol Sci. 2000;54:384-9.

62. Wang G, König R, Ansari GA, Khan MF. Lipid peroxidationderived aldehyde-protein adducts contribute to trichloroethenemediated autoimmunity via activation of CD4+ T cells. Free Radic Biol Med. 2008;44:1475-82.

63. Wang G, Wang J, Ma H, Ansari GA, Khan MF. N-acetylcysteine protects against trichloroethene-mediated autoimmunity by attenuating oxidative stress. Toxicol Appl Pharmacol. 2013;273:189-95.

64. Halmes NC, McMillan DC, Oatis Jr JE, Pumford NR. Immunochemical detection of protein adducts in mice treated with trichloroethylene. Chem Res Toxicol. 1996;9:451-6.

65.• US Environmental Protection Agency. IRIS Profile for Trichloroethylene. Available at: http://www.epa.gov/iris/subst/ 0199.htm. Accessed 22 April 2014. This site documents USEPA dose-response analysis of TCE-induced immune effects.

66. Gilbert KM, Rowley B, Gomez-Acevedo H, Blossom SJ. Coexposure to mercury increases immunotoxicity of trichloroethylene. Toxicol Sci. 2011;119:281-92.

67. Winter WE, Schatz DA. Autoimmune markers in diabetes. Clin Chem. 2011;57:168-75.

68. Castro C, Gourley M. Diagnostic testing and interpretation of tests for autoimmunity. J Allergy Clin Immunol. 2010;125(2 Suppl 2): S238-47.

69. Kopf PG, Scott JA, Agbor LN, Boberg JR, Elased KM, Huwe JK, et al. Cytochrome P4501A1 is required for vascular dysfunction and hypertension induced by 2,3,7,8-tetrachlorodibenzo-p-dioxin. Toxicol Sci. 2010;117:537-46.

70. Sultana S, Kolla VK, Peddireddy V, Jeedigunta Y, Penagaluru PK, Joshi S, et al. Association of CYP1A1 gene polymorphism with ischemic stroke in South Indian population. Transl Stroke Res. 2011;2:26-32.

71. Marinković N, Pasalić D, Potocki S. Polymorphisms of genes involved in polycyclic aromatic hydrocarbons biotransformation and atherosclerosis. Biochem Med (Zagreb). 2013;23:255-65.

72. Senft AP, Dalton TP, Nebert DW, Genter MB, Puga A, Hutchinson $\mathrm{RJ}$, et al. Mitochondrial reactive oxygen production is dependent on the aromatic hydrocarbon receptor. Free Radic Biol Med. 2002;33: $1268-78$. 
73. Zhang N, Agbor LN, Scott JA, Zalobowski T, Elased KM, Trujillo A, et al. An activated renin-angiotensin system maintains normal blood pressure in aryl hydrocarbon receptor heterozygous mice but not in null mice. Biochem Pharmacol. 2010;80:197-204.

74. Ilhan S, Atessahin D, Atessahin A, Mutlu E, Onat E, Sahna E. 2,3,7, 8-tetrachlorodibenzo-p-dioxin-induced hypertension: the beneficial effects of melatonin. Toxicol Ind Health. 2013.

75. Jules GE, Pratap S, Ramesh A, Hood DB. In utero exposure to benzo(a)pyrene predisposes offspring to cardiovascular dysfunction in later-life. Toxicology. 2012;295:56-67.

76. Chahin A, Peiffer J, Olry JC, Crepeaux G, Schroeder H, Rychen G, et al. EROD activity induction in peripheral blood lymphocytes, liver and brain tissues of rats orally exposed to polycyclic aromatic hydrocarbons. Food Chem Toxicol. 2013;56:371-80.

77. Cicchetti R, Argentin G. The role of oxidative stress in the in vitro induction of micronuclei by pesticides in mouse lung fibroblasts. Mutagenesis. 2003;18:127-32.

78. Godderis L, Aka P, Mateuca R, Kirsch-Volders M, Lison D, Veulemans H. Dose-dependent influence of genetic polymorphisms on DNA damage induced by styrene oxide, ethylene oxide and gamma-radiation. Toxicology. 2006;219:220-9.

79. Sinitsky MY, Druzhinin VG. The application of the cytokinesisblock micronucleus assay on peripheral blood lymphocytes for the assessment of genome damage in long-term residents of areas with high radon concentration. J Radiat Res. 2014;55:61-6.

80. Nair-Shalliker V, Fenech M, Forder PM, Clements MS, Armstrong BK. Sunlight and vitamin D affect DNA damage, cell division and cell death in human lymphocytes: a cross-sectional study in South Australia. Mutagenesis. 2012;27:609-14.

81. Benassi-Evans B, Fenech M. Chronic alcohol exposure induces genome damage measured using the cytokinesis-block micronucleus cytome assay and aneuploidy in human B lymphoblastoid cell lines. Mutagenesis. 2011;26:421-9.

82. Fenech $\mathrm{M}$, Bonassi $\mathrm{S}$. The effect of age, gender, diet and lifestyle on DNA damage measured using micronucleus frequency in human peripheral blood lymphocytes. Mutagenesis. 2011;26:43-9.

83. Bonassi S, Znaor A, Ceppi M, Lando C, Chang WP, Holland N, et al. An increased micronucleus frequency in peripheral blood lymphocytes predicts the risk of cancer in humans. Carcinogenesis. 2007;28:625-31.

84. Cakmak DG, Aykanat B, Fidan K, Gulleroglu K, Bayrakci US, Sepici A, et al. Micronucleus frequencies in peripheral blood lymphocytes of children with chronic kidney disease. Mutagenesis. 2011;26:643-50.

85. Palazzo RP, Bagatini PB, Schefer PB, de Andrade FM, Maluf SW. Genomic instability in patients with type 2 diabetes mellitus on hemodialysis. Rev Bras Hematol Hemoter. 2012;34:31-5.

86.• Andreassi MG, Barale R, Iozzo P, Picano E. The association of micronucleus frequency with obesity, diabetes and cardiovascular disease. Mutagenesis. 2011;26:77-83. This study demonstrates the value of the micronucleus as a biochemical marker in predicting disease outcome in the general population. 